\section{Antioxidatives Potential von standardisiertem Myrtol bestätigt}

— Die antioxidative Wirkung von Myrtol standardisiert (GeloMyrtol ${ }^{\circledast}$ forte) wurde nun in einer aktuellen Untersuchung nochmals bestätigt: Der Wirkstoff reduziert die Freisetzung von Sauerstoffradikalen in alveolären Makrophagen von COPD-Patienten um ca. 18 Prozent. Die antioxidative Wirkung von Myrtol sei laut Rantzsch und Kollegen (Leipzig) somit vergleichbar mit der von Acetylcystein. Ebenfalls in vitro wurden von den Wissenschaftlern die anti-inflammatorischen Eigenschaften von Myrtol und weiteren ätherischen Ölen untersucht. Ergebnis war, dass Myrtol die Freisetzung der Entzündungsbotenstoffe TNF-a um $37 \%$ und GM-CSF um $36 \%$ hemmt.

In einer klinischen Studie zur chronischen Bronchitis konnte nachgewiesen werden, dass Myrtol standardisiert vor akuten Exazerbationen schützen kann. Die aktuellen In-vitro-Ergebnisse unterstützen nochmals die Ansicht, dass nicht nur die mukosekretolytischen Effekte von Myrtol standardisiert

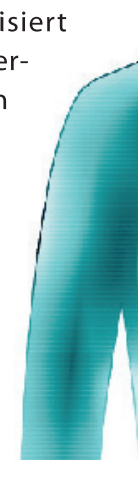

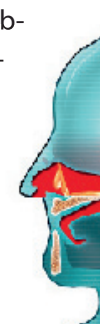

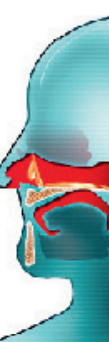

hierfür verantwortlich gemacht werden können, sondern ebenso wichtige Zusatzeffekte wie anti-inflammatorische und antioxidative Eigenschaften. Neben diesen Zusatzeffekten steigert GeloMyrtol ${ }^{\circledR}$ forte effektiv die ziliäre Schlagfrequenz. Mit diesem umfassenden pharmakologischen Wirkprofil liefert das Präparat überzeugende Ergebnisse bei der Behandlung der akuten und chronischen Sinusitis sowie der akuten und chronischen Bronchitis. Das zeigen 26 zum Teil GCP-konforme Studien an ca. 5800 Patienten. y

\section{Nach Information der
Pohl-Boskamp GmbH \\ Nach Information der
Pohl-Boskamp GmbH}

Meerwasserspülung mit Hyaluronsäure für Nase und Ohr

Seit Anfang des Jahres ist in Deutschland die hyaluronsäurehaltige Meerwasserspülung Aliamare ${ }^{\circledast}$ von IBSA (Institut Biochimique SA) erhältlich. Die Spülung kann gleichermaßen für Nase und Ohr eingesetzt werden. Im Bereich der Nase befreit sie effizient von überschüssigem Nasensekret, Pollen und Feinstaub und befeuchtet und pflegt gleichzeitig die Nasenschleimhaut. Das Meerwasser in Aliamare ${ }^{\varpi}$ verflüssigt den Schleim und reinigt. Die Hyaluronsäure ist ebenfalls eine natürlich Substanz und verfügt über ein starkes Wasserbindevermögen. In Spülungen kann sie die Wundheilung beschädigter Haut unterstützen und dazu beitragen, dass durch die Bildung eines Films auf der Schleimhaut eine zusätzliche Schicht liegenbleibt.

Im Ohr reinigt die Synthese aus Meerwasser und Hyaluronsäure die äußeren Gehörgänge, löst OhrenschmalzPfropfen auf und beugt deren neuerlicher Bildung vor. Gerade für Hörgeräteträger ist eine regelmäßige Pflege des Gehörgangs wichtig.

Nach Informationen von IBSA Institut Biochimique SA

Anschauliche Aufklärung für bessere Compliance

Die bene-Arzneimittel GmbH, Hersteller des lokal-physikalisch wirkenden und bewährten Mukolytikums Tacholiquin ${ }^{\oplus}$, entwickelt im Rahmen des kontinuierlich erweiterten Compliance-Kompendiums praktische Materialien, um Ärzte die Erklärung komplexer Zusammenhänge im Therapiegespräch zu erleichtern. Denn die beste Therapie hat keine Aussicht auf Erfolg wenn es an der Compliance mangelt. Nun hat bene-Arzneimittel seine Reihe der fachmedizinischen Tischaufsteller um einen dritten Schwerpunkt erweitert: Atemwegserkrankungen. Der Aufsteller ist so gestaltet, dass alle wesentlichen Schlüsselbegriffe auf der dem Arzt zugewandten Seite stehen, während auf der Patientenseite farbige Grafiken und einfache Erklärungen das Krankheitsbild und Behandlungsmöglichkeiten auf verständliche Weise erläutern. Ärzte können den neuen Aufsteller bei bene-Arzneimittel anfordern.

Nach Informationen der bene-Arzneimittel GmbH 